\title{
Underutilization of the Subcutaneous Route of Administration
}

\author{
Kenneth W Locke* \\ Senior Medical Director, USA \\ *Corresponding author: Kenneth W Locke, Senior Medical Director, Global Medical Affairs, Halozyme, San Diego, USA. \\ To Cite This Article: Kenneth W Locke. Underutilization of the Subcutaneous Route of Administration. Am J Biomed Sci \& Res. 2019 - 4(3). \\ AJBSR.MS.ID.000788. DOI: 10.34297/AJBSR.2019.04.000788
}

Received: : July 13, 2019 | Published: July 26, 2019

\section{Introduction}

Therapeutic proteins have become an important part of treatment, particularly for serious and chronic diseases, and the subcutaneous (SC) route of administration has become a more popular, yet still underutilized mode of delivery. The extracellular matrix of the hypodermis is composed of a network of fibrous proteins (e.g., collagen and elastin) embedded within a viscoelastic gel. The viscoelastic gel is composed of glycosaminoglycans and proteoglycans that create resistance to bulk fluid flow in the SC space. The primary glycosaminoglycan is the highly hydrated hyaluronan, a mega-dalton polymer of repeating disaccharide units of $\mathrm{N}$-acetylglucosamine and glucuronic acid. Following SC injection of water-soluble small molecules, absorption into the systemic circulation occurs via local capillaries. In contrast, large molecules (e.g., proteins) reach the systemic circulation via bulk fluid flow into draining lymphatic vessels. Regardless of the size of the injected molecule, current medical dogma suggests that rapid SC injections are limited to a volume of $\sim 2 \mathrm{~mL}$. Injection of volumes of $>2 \mathrm{~mL}$ are believed to be associated with injection site pain, adverse effects at the injection site (e.g., induration) and injection site leakage [1]. However, there is little evidence from controlled studies to substantiate these assumptions [2].

There are several strategies for overcoming volume restrictions to SC drug administration. Some biologics (e.g., adalimumab) can be concentrated to a sufficient degree to administer an adequate dose in a relatively low volume $(<2 \mathrm{~mL})$. Others (e.g., sekukinumab, SCIg) require multiple low volume injections, often across multiple sites, to deliver therapeutic doses. Recently, on-body devices have been developed that are capable of delivering greater volumes (e.g., $10-25 \mathrm{~mL}$ ) very slowly.

Enzymes that have a pharmacologic action to degrade the glycosaminoglycans in the SC space can function as permeation enhancers. Hyaluronidases, like the recombinant human hyaluronidase PH20 (rHuPH20) enzyme, when injected into the SC space, cleave hyaluronan into small oligomers of $\mathrm{N}$-acetylglucosamine and glucuronic acid, resulting in a reduction in the resistance to flow. By reducing resistance to flow, rHuPH20 removes the barriers to large volume SC drug delivery, dispersion, and absorption. Biologics once limited to intravenous (IV) administration because of the volumes involved, can be delivered SC when co-formulated or co-administered with rHuPH20. Administration time of these larger volumes can be reduced from hours to minutes. Examples of IV to SC switches include two anticancer therapies, trastuzumab (i.e., HERCEPTIN HYLECTATM/ Herceptin $^{\circledR}$ SC) and rituximab (i.e., RITUXAN HYCELA ${ }^{\circledR} /$ RITUXAN $^{\circledR}$ $\mathrm{SC} /$ MabThera ${ }^{\circledR} \mathrm{SC}$ ) that are co-formulated with $\mathrm{rHuPH} 20$ and human immunoglobulin (i.e., HyQvia ${ }^{\circledR} /$ HYQVIA $^{\circledR}$ ) that is dosed sequentially after rHuPH20 to treat primary immunodeficiency. Recently, as reported at the 2019 American Society of Clinical Oncology (ASCO) Annual Meeting, non-inferior efficacy and pharmacokinetics for the SC administered formulation of daratumumab (co-formulated with rHuPH20) compared to the approved IV formulation was demonstrated in patients with relapsed/refractory multiple myeloma. Administration time was reduced from a median of more than 3 hours for the IV formulation ( 7 hours for the first administration) to a median of 5 minutes for the SC formulation [3]. In addition, a lower rate of infusion-related reactions was observed in the arm that received the SC administered formulation of daratumumab compared to DARZALEX ${ }^{\circledR}$ IV (13 percent vs. 35 percent, respectively) [3].

Beyond increases in delivery volumes and shorter administration times, hyaluronidase permeation enhancers may also yield potential pharmacokinetic improvements (e.g., increased Cmax, shortened Tmax, increased bioavailability, reduced pharmacokinetic variability) compared to SC formulations without the enzyme to optimize existing SC formulations or to facilitate the development of SC formulations of novel therapeutics [3,4]. Because chronic diseases require frequent and long-term treatment, the $\mathrm{SC}$ route of administration may offer both a time- and cost-saving alternative to IV administration. SC administration offers the potential for patient self-administration at home. Even for the SC therapeutics that must still be administered in a health care facility, 
both time- and cost-savings may be realized. For example, the SC formulation of trastuzumab has been shown to reduce both patient chair and active healthcare provider times versus IV infusion [5]. Moreover, in a UK study, cost savings associated with SC versus IV trastuzumab have been estimated at $£ 111.81$ per patient episode, equating to a potential saving of $£ 2012.58$ per patient over a full course of adjuvant trastuzumab treatment for HER2-positive early breast cancer [6]. In addition, patients have indicated a significant preference for SC trastuzumab over IV infusion, whether delivered via a single-use injection device or a "hand-held" syringe [7].

The SC route of administration is currently underutilized. SC administration, particularly when combined with permeation enhancers, may enable the delivery of protein therapeutics to overcome administration, time, and volume barriers associated with traditional SC administration and to provide meaningful benefits to patients and health care providers compared to IV administration.

\section{References}

1. Frost G (2007) Recombinant human hyaluronidase (rHuPH20): an enabling platform for subcutaneous drug and fluid administration. Expert Opin Drug Deliv 4(4): 427-440.
2. Mathaes R, Koulov A, Joerg S, Mahler HC (2016) Subcutaneous injection volume of biopharmaceuticals-Pushing the boundaries. J Pharm Sci 105(8): 2255-2259.

3. Maria Victoria Mateos, Hareth Nahi, Wojciech Legiec, Sebastian Grosicki, Vladimir Vorobyev, et al. (2019) Efficacy and Safety of the Randomized, Open-Label, Non-inferiority, Phase 3 Study of Subcutaneous (SC) Versus Intravenous (IV) Daratumumab (DARA) Administration in Patients (pts) With Relapsed or Refractory Multiple Myeloma (RRMM): COLUMBA. An American Society of Clinical Oncology Journal.

4. Locke KW, Maneval DC, LaBarre MJ (2019) ENHANZE® drug delivery technology: a novel approach to subcutaneous administration using recombinant human hyaluronidase PH20. Drug Deliv 26(1): 98-106.

5. De Cock E, Pivot X, Hauser N, Verma S, Kritikou P, et al. (2016) A time and motion study of subcutaneous versus intravenous trastuzumab in patients with HER2-positive early breast cancer. Cancer Med 5(3): 389397.

6. Russell Burcombe, Steve Chan, Richard Simcock, Kunal Samanta, Fran Percival, et al. (2013) Subcutaneous trastuzumab (Herceptin $®$ ): a UK time and motion study in comparison with intravenous formulation for the treatment of patients with HER2-positive early breast cancer. Advances in Breast Cancer Research 2(4):133-140.

7. Pivot X, Gligorov J, Müller V, Curigliano G, Knoop A, et al. (2014) Patients' preferences for subcutaneous trastuzumab versus conventional intravenous infusion for the adjuvant treatment of HER2-positive early breast cancer: final analysis of 488 patients in the international, randomized, two-cohort PrefHer study. Ann Oncol 25(10): 1979-1987. 